\title{
Initiating buprenorphine/naloxone for opioid use disorder in the emergency department
}

\section{Keysha Low}

\begin{abstract}
Opioid use disorder (OUD) is a public health crisis that continues to affect individuals across Canada, and requires a multifaceted approach to minimize its impact. Through the chronic consumption of opioids, many individuals can become dependent on opioids and develop OUD. Buprenorphine/naloxone is the recommended first-line treatment, whenever possible, for patients living with OUD. The Emergency Strategic Clinical Network ${ }^{\mathrm{TM}}$ within Alberta Health Services is targeting the crisis in emergency departments by implementing a provincially standardized program. The Buprenorphine/ Naloxone Initiations in Emergency Departments program includes identifying OUD, initiating treatment with the medication buprenorphine/naloxone, and providing rapid and reliable referrals to community clinics for titration and continued patient care. This paper provides an overview of opioids, OUD, opioid agonist treatments such as buprenorphine/naloxone, and details the program and protocol available in Alberta.
\end{abstract}

Keywords: opioids, opioid use disorder (OUD), opioid agonist treatment (OAT), buprenorphine/naloxone, emergency department (ED)

\section{Background}

$\mathrm{T}$ he opioid crisis continues to affect Canadians of various ages, genders, geographical locations, and socioeconomic statuses. From 2016 to 2019, there were over 13,900 deaths related to opioids in Canada (Government of Canada, 2019). In 2018 alone, there were 4,460 documented apparent opioid-related deaths, equating to one life lost an average of every 2 hours (Government of Canada, 2019). Multiple factors have contributed to the current crisis. From the late 1990s to the early 2000s, certain pharmaceutical companies encouraged healthcare providers to treat patients with opioids, suggesting that they were highly effective and non-addictive (Jones et al., 2018). This resulted in a significant rise in the rate of opioid prescribing in Canada. Along with increasing prescribing rates, has come increasing rates of opioid misuse, abuse, and overdoses. In response, many healthcare providers have tried to reduce or stop prescribing opioids. When patients are no longer able to obtain prescribed opioids, many turn to illicit opioids to manage their pain and/or dependence. Over the last decade, a surge in illicit substances that contain synthetic opioids has further amplified the crisis. These illicit substances often contain unregulated levels of potent opioids, such as fentanyl, making them extremely dangerous. That is, they present a high risk of overdose.

In recent years, Canada has focused on implementing policies and strategies to address this public health crisis. These include legislative and regulatory changes to increase awareness and knowledge among healthcare professionals, increase access to treatment and harm reduction strategies, and increase availability and distribution of naloxone to the public for reversing overdoses (Health Canada, 2017, 2019). At the same time, there has been increasing efforts to enforce laws and regulations that focus on eliminating the manufacturing, distribution, and trafficking of illegal opioids into and within Canada (Health Canada, 2019). Despite these efforts, the rate and the number of overdoses in Canada continues to rise each year.

Many jurisdictions across the country have implemented local initiatives to respond to the opioid crisis. In Alberta, the Emergency Strategic Clinical Network ${ }^{\text {тx }}$ (ESCN) within Alberta Health Services (AHS) has been leading the implementation of a province-wide strategy to help address the opioid crisis in emergency departments and urgent care centres (EDs) since March 2018. The ESCN is a group of healthcare professionals and patients working together across the province to improve emergency care and services in Alberta. The ESCN created a provincial expert working group that included physician experts, community/primary care services, and addictions and mental health experts. The team developed a province-wide initiative that starts treatment for individuals living with opioid use disorder (OUD) during an ED visit. The Buprenorphine/Naloxone Initiations in Emergency Departments program includes identifying OUD, initiating treatment with the medication buprenorphine/naloxone, and providing rapid and reliable referrals to community clinics for titration and continued patient care.

\section{Opioids}

Naturally found in the opium poppy (Papaver somniferum), opioids are manufactured as medications for pain management. Opioids are a class of substances that act on the central nervous system in the same way that endorphins operate through the activation of the opioid receptors mu, kappa and delta. Activation of these opioid receptors inhibits excitability and pain neurotransmission, which results in analgesic and possibly euphoric effects. These receptors also increase dopamine production and may influence the brain reward system. This natural reward system 
can cause individuals to repeat compulsive behaviours in order to replicate the dopamine release response. Chronic consumption of opioids may result in a chemical rewiring of the brain that leads to tolerance (needing to have higher doses to achieve the same effect), and physical dependence on opioids. Individuals who use opioids chronically may experience terrible physical and psychological craving and withdrawal symptoms when discontinued (National Institutes of Health, 2019).

\section{Opioid Use Disorder}

OUD is a chronic relapsing illness that involves a problematic pattern of opioid use. OUD includes the misuse of prescribed opioid medications, use of diverted prescription opioids, or use of illicitly manufactured opioids (Canadian Research Initiative on Substance Misuse (CRISM), 2018). The current Canadian national guideline recommends evidence-based long-term treatment of OUD using opioid agonist treatment (OAT) with or without psychosocial treatment (CRISM, 2018). The guideline also identifies buprenorphine/naloxone as the recommended first-line OAT for OUD wherever feasible.

OAT is significantly more effective than non-pharmacological treatments, and there is strong evidence that OAT effectively reduces morbidity, mortality, and the risk of HIV and hepatitis $\mathrm{C}$ infections among individuals that inject substances (CRISM, 2018). Withdrawal management alone without OAT will reduce a patient's level of opioid tolerance. This is not advisable due to the high rates of relapse that can lead to increased risk of morbidity and mortality (Bruneau et al., 2018; CRISM, 2018). Compared to other OAT medications such as methadone, buprenorphine/naloxone has many advantages. These include fewer side effects and lower risk of toxicity and drug-drug interactions (Bonhomme, Shim, Gooden, Tyus \& Rust, 2012; Moody, 2013). Buprenorphine/naloxone allows for greater flexibility and patient autonomy, which results in increased treatment compliance, retention, and reduced substance use (CRISM, 2018; Holland et al., 2014).

\section{Buprenorphine/Naloxone}

Buprenorphine/naloxone is a 4:1 ratio combination medication generally taken once a day when patients are on stable maintenance doses. Buprenorphine is a long-acting partial opioid agonist that has a half-life of approximately 24-42 hours (CRISM, 2018). As a partial opioid agonist, buprenorphine provides little to no euphoric effects due to having lower intrinsic activity on opioid receptors than full opioid agonists (for example, fentanyl, morphine, heroin, etc.). When buprenorphine has fully saturated the receptors, it achieves a ceiling effect and additional doses will not increase its effectiveness. This ceiling effect reduces overdose and respiratory depression potential, making it a safer option. In addition to its high safety profile, buprenorphine has a high affinity for the receptor that will out-compete and can even displace other full agonist opioids (National Alliance of Advocates for Buprenorphine Treatment, 2019). Naloxone is an opioid antagonist that has poor bioavailability when administered sublingually or orally. It is included in the medication to lower its abuse potential (Drugbank, 2019). If the medication is diverted and injected, a sufficient dose of naloxone will be absorbed and induce withdrawal symptoms.

\section{Buprenorphine/Naloxone in Emergency Departments}

Buprenorphine/naloxone has been in Canada since 2007 but was typically only available in addiction medicine outpatient clinic settings. The ESCN recognized a potential opportunity to help patients by having the medication available in the ED. Due to the rising number of ED opioid-related presentations in Alberta, EDs provide an effective opportunity to intervene and initiate treatment that addresses the underlying condition of OUD, going beyond the extremely important practice of offering naloxone kits. Emergency departments are portals for identifying patients who live with OUD, who may present with an overdose, other concerns related to opioids, or issues not directly related to addictions (Winetsky, Weinrieb \& Perrone, 2017). In 2018, 13\% of individuals in Alberta with an apparent accidental death related to fentanyl had presented to an ED within 30 days before dying, highlighting the ED opportunity to intervene and the potential to prevent a catastrophic event (Government of Alberta, 2019). ED patients are more likely to continue engaging in treatment if they initiate buprenorphine/naloxone in the ED. In a key study, 78\% (89/114) of ED patients randomized to buprenorphine/naloxone treatment remained in treatment 30 days later, compared to $37 \%$ (38/102) of patients who were referred to treatment without initiating buprenorphine/naloxone (D’Onofrio et al., 2015).

The Buprenorphine/Naloxone Initiations in Emergency Departments program was the first of its kind in Canada with a provincial scope. The ESCN supported the training and implementation of the standardized treatment protocol for patients who present with OUD in all adult EDs across Alberta. The ESCN team collaborated with multidisciplinary teams at each site, addressed various local needs and concerns, developed local champions, and provided expert front-line education and resources to more than two thousand emergency nurses, staff and physicians. Each local team typically included site and unit managers, emergency and primary care physicians, clinical nurse educators, pharmacists, social workers, and front-line emergency nurses. These local champions established program and protocol expertise, which they use to guide their colleagues on a day-to-day basis to ensure program sustainability over time.

There are programs in other jurisdictions across the country that offer buprenorphine/naloxone and each clinical pathway may vary in dosing, assessments, and treatment options. In the current AHS provincial ED program, there are three key steps (Figure 1):

1. Identify OUD;

2. Initiate buprenorphine/naloxone for eligible patients in or out of the ED; and

3. Provide a rapid community clinic referral for medication titration and continued patient care.

\section{Step 1: Identify Opioid Use Disorder}

To be eligible for buprenorphine/naloxone, patients should meet the American Psychiatric Association (2013) Diagnostic and Statistical Manual of Mental Disorders, Fifth Edition, (DSM5) diagnostic criteria for OUD. In clinical practice, ED nurses can easily apply the " $4 \mathrm{Cs}$ " of addiction to help identify patients 


\section{Alberta Health Services Provincial Program}
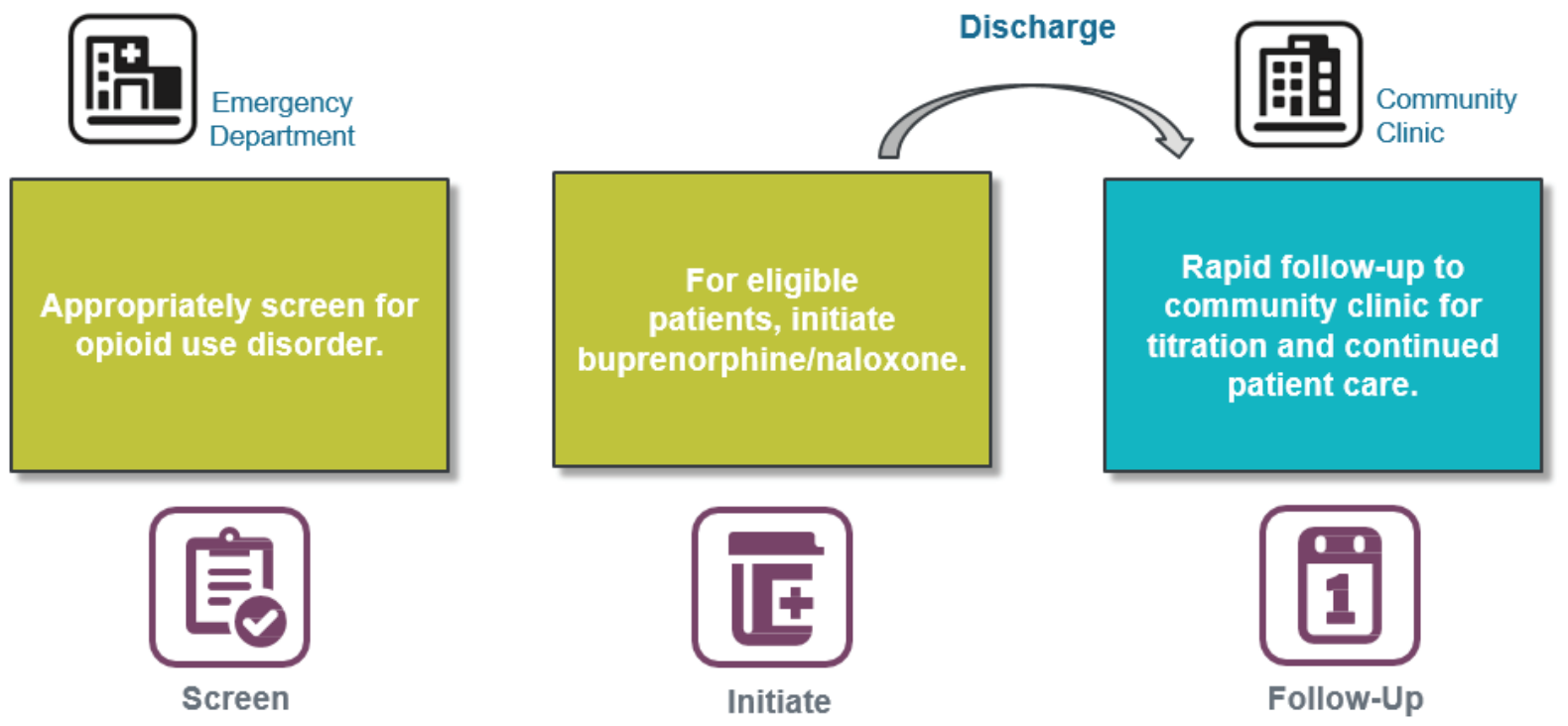

Figure 1. Three key steps in the Buprenorphine/Naloxone Initiations in Emergency Departments program.

with addiction to any substance, including opioids. Problematic opioid use can be recognized by asking patients about craving, loss of control of amount or frequency of use, compulsion to use, and use despite consequences (Centre for Addiction and Mental Health, 2019). After the identification of OUD, patients may need encouragement and education from ED healthcare professionals to consider treatment. The ED nurse plays a vital role in establishing a safe and open environment where the patient feels respected and understood in a way that has the potential to motivate change. It is important to view every patient interaction as an opportunity to engage, educate, and offer supports. Some patients may not be ready to make challenging life changes during their ED visit and may need more time to make that decision.

\section{Step 2: Initiate Buprenorphine/Naloxone in or out of the Emergency Department}

The next step is to initiate buprenorphine/naloxone in or out of the ED. There is absolute criteria listed in the protocol that patients need to meet (suspicion of OUD, willingness to engage in the medication, and no allergy to buprenorphine or naloxone) before safely receiving the medication. The protocol also identifies relative contraindications for specific patient populations that may benefit from expert consultation prior to initiation, such as pregnant or adolescent patients. If patients meet the absolute criteria, clinicians need also to consider the appropriate timing of initiation, where patients should be in a moderate level of withdrawal before receiving their first dose (Figure 2). Using the Clinical Opiate Withdrawal Scale (COWS) (Wesson \& Ling, 2003) assessment tool, nurses are able to assess the patient's level of withdrawal quickly and objectively by finding the sum of 11 criteria (see Appendix A). If the score is 12 or greater, patients qualify for ED induction, receiving initial doses of buprenorphine/naloxone in the ED one hour apart, and is then discharged with additional doses to take at home along with patient teaching and a take home naloxone kit (see Appendix B).

If the score is less than 12, patients may still have another opioid on their receptors and would qualify for home induction. Due to the high affinity of buprenorphine, taking the medication too soon displaces the full opioid agonist and precipitates withdrawal symptoms, making the patient feel worse. In order to avoid precipitated withdrawal, patients are discharged with the instructions to take their doses at home once they start to experience moderate withdrawal symptoms and/or cravings. Home induction patients also receive teaching and a take home naloxone kit prior to discharge (see Appendix C).

\section{Step 3: Provide Rapid Community Clinic Referral for Continued Patient Care}

Patients initiating buprenorphine/naloxone usually require titration over two to three days to reach a therapeutic dose. In this protocol, the ED completes day one of the titration, followed by a rapid referral to a community clinic and pharmacy that will follow up the next day for continued medication titration and patient care. Through collaboration with local community resources and pharmacies, there are several guaranteed next day walk-in referral options for patients, which include primary care, brick-and-mortar OUD treatment clinics, and virtual medicine options available across Alberta. As a system, these clinics ensure that Albertans anywhere in the province can receive OAT. 


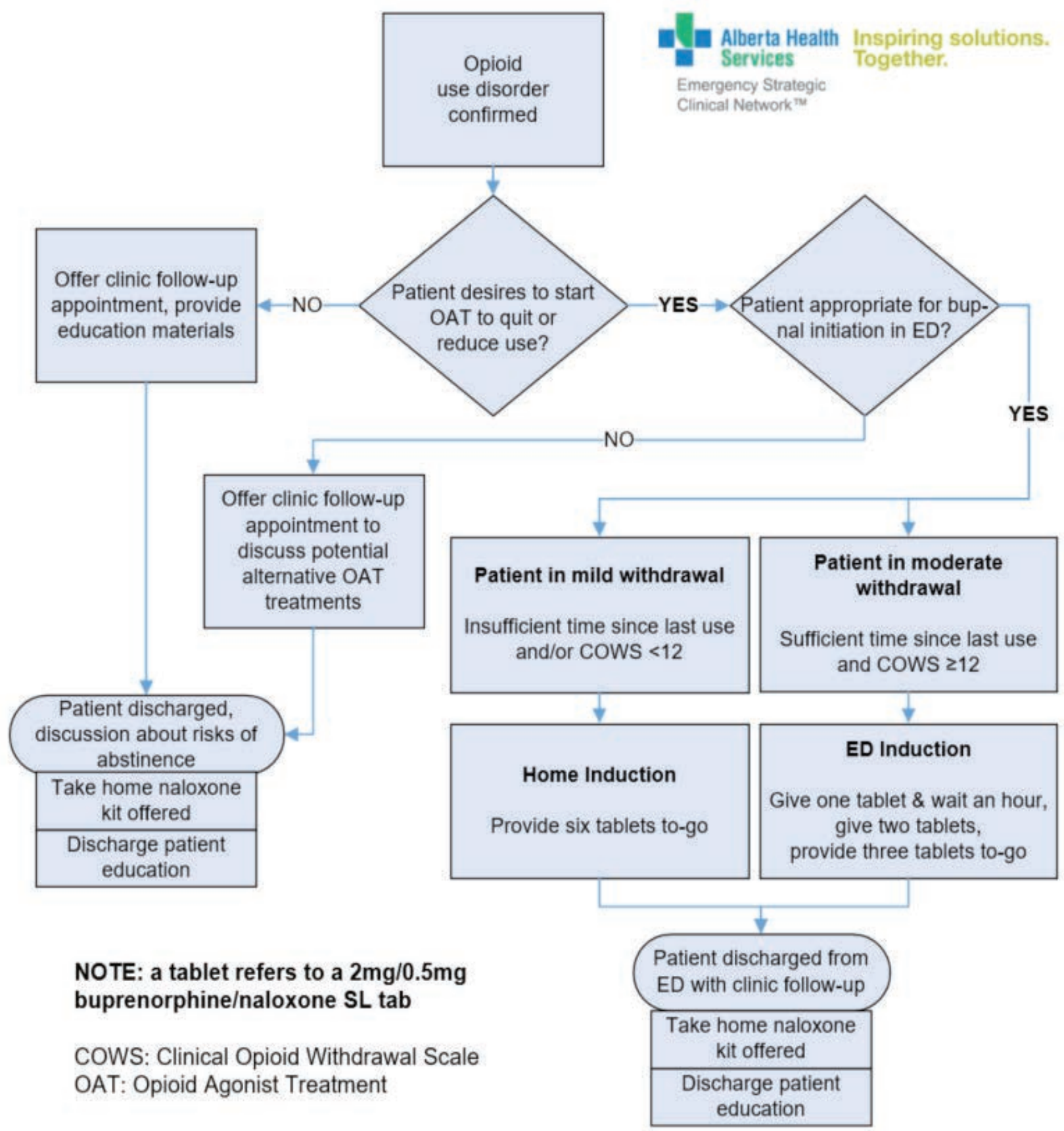

Figure 2. Standardized buprenorphine/naloxone initiation protocol in all adult EDs across Alberta.

\section{Future of the Opioid Crisis}

At the time of publication, the ESCN has helped over 100 sites implement the protocol and is currently supporting the remaining sites to complete program implementation by early 2020 (see Appendix D). Identifying OUD and starting patients on buprenorphine/naloxone is not difficult or time intensive. The healthcare system as a whole in Alberta has made significant efforts to bring the medication to a variety of settings, such as correctional facilities, homeless shelters, and primary care. The province has also been working on improving accessibility and support for Indigenous patients and higher risk populations.
Although the future of the opioid crisis is unknown, it is predicted to continue over the coming years (Chen et al., 2019). While the crisis is complex and requires a multifaceted approach, providing patients with easy access to buprenorphine/naloxone can have significant effects in limiting the crisis across the country. Emergency nurses play a key role throughout every patient's visit, and influence each step of the OUD treatment protocol. ED nurses across Canada are encouraged to advocate for patients living with OUD and support the effective initiation and use of buprenorphine/naloxone. We should not overlook this valuable opportunity to prevent deaths related to OUD and to help our patients stabilize their lives, be safe and stay healthy. 


\section{Declarations}

I, Keysha Low, am employed by AHS. My current position is funded by Alberta Health through the Minister's Opioid Emergency Response Commission (MOERC). The funder did not direct the contents of this manuscript.

\section{Acknowledgements}

The author would like to thank Catherine Biggs BScPharm, Scott

Fielding RN MBA, Heather Hair RN MBA, Patrick McLane MA
PhD, Heli Mehta MHI, Ken Scott MA MBA, and Lee Zorniak RN $B S c N$ for their contributions.

\section{About the Author}

Keysha Low RN BN MHA CHE has worked as an emergency nurse in a variety of remote, rural and large tertiary care centres across the U.S. and Canada. She currently works as a Senior Consultant with the Emergency Strategic Clinical Network ${ }^{\mathrm{TM}}$ and as an emergency nurse at the Foothills Medical Centre in Calgary, $A B$.

\section{References}

American Psychiatric Association. (2013). Diagnostic and Statistical Manual of Mental Disorders, Fifth Edition. Arlington, VA: American Psychiatric Publishing, Inc.

Bonhomme J., Shim, R., Gooden, R., Tyus, D. \& Rust, G. (2012). Opioid addiction and abuse in primary care practice: A comparison of methadone and buprenorphine as treatment options. Journal of the National Medical Association 104(0): 342-350.

Bruneau J., Ahamad, K., Goyer, M-E., Poulin, G., Selby, P., Fischer, B., Wild, C. \& Wood, E. (2018). Management of opioid use disorders: A national clinical practice guideline. Canadian Medical Association Journal, 190(9): E247-E257. http://www.cmaj.ca/ content/cmaj/190/9/E247.full.pdf

Canadian Research Initiative on Substance Misuse. (2018). CRISM National Guideline for the Clinical Management of Opioid Use Disorder. https://crism.ca/wp-content/uploads/2018/03/ CRISM_NationalGuideline_OUD-ENG.pdf.

CentreforAddictionandMentalHealth.(2019).Addiction.https://www. camh.ca/en/health-info/mental-illness-and-addiction-index/ addiction

Chen, Q. Larochelle, M., Weaver, D., Lietz, A., Mueller, P., Mercaldo, S...Chhatwal, J. (2019). Prevention of prescription opioid misuse and projected overdose deaths in the United States. Journal of the American Medical Association Network Open 2(2): e187621.

D’Onofrio, G., O'Connor, P., Pantalon, M., Chawarski, M., Busch, S., Owens, P., Bernstien, S. \& Fiellin, D. (2015). Emergency department-initiated buprenorphine/naloxone treatment for opioid dependence: A randomized clinical trial. Journal of American Medical Association, 313(16): 1636-1644.

Drugbank. (2019). Naloxone. https://www.drugbank.ca/drugs/ DB01183

Government of Alberta. (2019). Alberta Opioid Response Surveillance Report: Q2 2019. https://www.alberta.ca/opioid-reports.aspx.

Government of Canada. (2019). National report: Apparent opioidrelated deaths in Canada. https://health-infobase.canada.ca/ datalab/national-surveillance-opioid-mortality.html
Health Canada. (2017). Government of Canada: Actions on Opioids. https://www.canada.ca/content/dam/hc-sc/documents/ services/publications/healthy-living/actions-opioids-20162017/Opioids-Response-Report-EN-FINAL.pdf

Health Canada. (2019). Government of Canada Federal Actions on Opioids. https://www.canada.ca/content/dam/hc-sc/ documents/services/substance-use/problematic-prescriptiondrug-use/opioids/responding-canada-opioid-crisis/federalactions/federal-actions-overview.pdf

Holland R., Maskrey, V., Swift, L., Notley, C., Robinson, A., Nagar, J., Gale, T. \& Kouimtsidis, C. (2014). Treatment retention, drug use and social functioning outcomes in those receiving 3 months versus 1 month of supervised opioid maintenance treatment: Results from the super c randomized controlled trial. Addiction, 109(4): 596-607.

Jones, M., Viswanath, O., Peck, J., Kaye, A., Gill, J. \& Simopoulos, T. (2018). A brief history of the opioid epidemic and strategies for pain medicine. Pain and Therapy, 7(1): 13-21.

Moody, D. (2013). Metabolic and toxicological considerations of the opioid replacement therapy and analgesic drugs: Methadone and buprenorphine. Expert Opinion on Drug Metabolism \& Toxicology, 9(6): 675-697.

National Alliance of Advocates for Buprenorphine Treatment. (2019). Thorough Technical Explanation of Buprenorphine. https://www. naabt.org/education/technical_explanation_buprenorphine.cfm

National Institutes of Health: U.S. National Library of Medicine. (2019). Opioid Addiction. https://ghr.nlm.nih.gov/condition/ opioid-addiction\#

Winetsky, D., Weinrieb, R. \& Perrone, J. (2017). Expanding Treatment Opportunities for Hospitalized Patients with Opioid Use Disorders. Perspectives in Hospital Medicine, Oct 2017, E1-E3.

Wesson, D. \& Ling, W. (2003). The Clinical Opiate Withdrawal Scale (COWS). Journal of Psychoactive Drugs, 35(2): 253-259, DOI: 10.1080/02791072.2003.10400007 
Appendix A: Clinical Opiate Withdrawal Scale (COWS) Tool

The COWS assessment tool used to identify the patient's level of opioid withdrawal.

\section{Alberta Health
Services}

\section{Clinical Opiate Withdrawal Scale}

For each item, check $(\checkmark)$ the number that best describes the patient's signs or symptom. Rate on just the apparent relationship to opiate withdrawal. For example, if heart rate is increased because the patient was jogging just prior to assessment, the increase pulse rate would not add to the score.

\begin{tabular}{|c|c|c|}
\hline Patient's First Name & Date (yyyy-Mon-dd) & Time (24hrs) \\
\hline \multicolumn{3}{|l|}{ Reason for this assessment } \\
\hline $\begin{array}{l}\text { Resting Pulse Rate: } \quad \text { beats/minute } \\
\text { Measured after patient is sitting or lying for one minute } \\
\square 0 \text { pulse rate } 80 \text { or below } \\
\square 1 \text { pulse rate } 81-100 \\
\square 2 \text { pulse rate } 101-120 \\
\square 4 \text { pulse rate greater than } 120\end{array}$ & \multicolumn{2}{|c|}{$\begin{array}{l}\text { GI Upset: over last } 1 / 2 \text { hour } \\
\square 0 \text { no Gl symptoms } \\
\square 1 \text { stomach cramps } \\
\square 2 \text { nausea or loose stool } \\
\square 3 \text { vomiting or diarrhea } \\
\square 5 \text { multiple episodes of diarrhea or vomiting }\end{array}$} \\
\hline $\begin{array}{l}\text { Sweating: over past } 1 / 2 \text { hour not accounted for by room temp } \\
\text { or patient activity. } \\
\square 0 \text { no report of chills or flushing } \\
\square 1 \text { subjective report of chills or flushing } \\
\square 2 \text { flushed or observable moistness on face } \\
\square 3 \text { beads of sweat on brow or face } \\
\square 4 \text { sweat streaming off face }\end{array}$ & \multicolumn{2}{|c|}{$\begin{array}{l}\text { Tremor observation of outstretched hands } \\
\square 0 \text { no tremor } \\
\square 1 \text { tremor can be felt, but not observed } \\
\square 2 \text { slight tremor observable } \\
\square 4 \text { gross tremor or muscle twitching }\end{array}$} \\
\hline $\begin{array}{l}\text { Restlessness Observation during assessment } \\
\square 0 \text { able to sit still } \\
\square 1 \text { reports difficulty sitting still, but is able to do so } \\
\square 3 \text { frequent shifting or extraneous movements of legs/ } \\
\quad \text { arms } \\
\square 5 \text { unable to sit still for more than a few seconds }\end{array}$ & \multicolumn{2}{|c|}{$\begin{array}{l}\text { Yawning Observation during assessment } \\
\square 0 \text { no yawning } \\
\square 1 \text { yawning once or twice during assessment } \\
\square 2 \text { yawning three or more times during } \\
\quad \text { assessment } \\
\square 4 \text { yawning several times/minute } \\
\end{array}$} \\
\hline $\begin{array}{l}\text { Pupil size } \\
\square 0 \text { pupils pinned or normal size for room light } \\
\square 1 \text { pupils possibly larger than normal for room light } \\
\square 2 \text { pupils moderately dilated } \\
\square 5 \text { pupils so dilated that only the rim of the iris is visible }\end{array}$ & \multicolumn{2}{|c|}{$\begin{array}{l}\text { Anxiety or Irritability } \\
\square 0 \text { none } \\
\square 1 \text { patient reports increasing irritability or } \\
\quad \text { anxiousness } \\
\square 2 \text { patient obviously irritable or anxious } \\
\square 4 \text { patient so irritable or anxious that } \\
\text { participation in the assessment is difficult }\end{array}$} \\
\hline $\begin{array}{l}\text { Bone or Joint aches if patient was having pain previously, only } \\
\text { the additional component attributed to opiates withdrawal is scored } \\
\square 0 \text { not present } \\
\square 1 \text { mild diffuse discomfort } \\
\square 2 \text { patient reports severe diffuse aching of joints/muscles } \\
\square 4 \text { patient is rubbing joints or muscles and is unable to sit } \\
\text { still because of discomfort }\end{array}$ & \multicolumn{2}{|c|}{$\begin{array}{l}\text { Gooseflesh skin } \\
\square 0 \text { skin is smooth } \\
\square 3 \text { piloerrection of skin can be felt or hairs } \\
\text { standing up on arms } \\
\square 5 \text { prominent piloerrection }\end{array}$} \\
\hline $\begin{array}{l}\text { Runny nose or tearing Not accounted for by cold symptoms or } \\
\text { allergies } \\
\square 0 \text { not present } \\
\square 1 \text { nasal stuffiness or unusually moist eyes } \\
\square 2 \text { nose running or tearing } \\
\square 4 \text { nose constantly running or tears streaming down } \\
\quad \text { cheeks }\end{array}$ & \multicolumn{2}{|c|}{$\begin{array}{l}\text { Total Score } \\
\text { Total score is the sum of all } 11 \text { items } \\
\text { erson completing assessment: }\end{array}$} \\
\hline
\end{tabular}

Score: 5-12 = mild; 13-24 = moderate; 25-36 = moderately severe; more than 36 = severe withdrawal $20900(2017-08)$ 


\section{Emergency Department Buprenorphine/Naloxone (Suboxone)}

\section{Instructions for Leaving the Hospital}

The Emergency Department gave you buprenorphine/naloxone (Suboxone) to help treat an opioid use disorder. You were given 3 more doses of buprenorphine/naloxone to take with you.

It's important that you keep taking this medicine. Congratulations on taking this step!

\section{What is buprenorphine/naloxone and how does it work?}

Buprenorphine/naloxone (Suboxone) is a pill that has 2 medicines. The goal of this medicine is to help you use other opioids less, so you are safer and healthier.

Buprenorphine is a type of opioid (like morphine, heroin or methadone) but it works differently than other types of opioids. Buprenorphine helps treat withdrawal and can get rid of cravings more safely than other medicines. Once you get the right dose of buprenorphine, it will prevent withdrawal symptoms and can help you stop taking other prescription or purchased opioids.

Naloxone (Narcan) is added to buprenorphine to prevent people from injecting it. When the pill is dissolved under the tongue, the naloxone doesn't do anything. If the pill is injected, the naloxone can cause withdrawal symptoms or prevent opioids from working.

\section{How do I take buprenorphine/naloxone?}

Take buprenorphine/naloxone by mouth and dissolve it under your tongue. This medicine may take 15 to 30 minutes to dissolve. It's important to dissolve this medicine under your tongue as it won't work if you swallow it. After you take the medicine, wait at least 30 minutes before you eat or drink.

\section{When should I take buprenorphine/naloxone at home?}

Take this medicine only when you are in moderate withdrawal. This is the point when you feel like you want to use or inject to prevent more withdrawal symptoms.

It's important that you feel really sick before you take your first dose of buprenorphine/naloxone. If you take it before you feel really sick, it can make your symptoms much worse.

\section{Dosing}

Each dose of buprenorphine/naloxone is $2 \mathrm{mg}$. Be sure to wait at least 1 hour in between doses. Record the time you take each dose on a piece of paper or in the notes app on your phone.

Don't take your next dose if you feel worse, sleepy, sedated, or have a hard time focusing. If this happens, call the clinic as your dose may be too strong. 
You should feel a little better or about the same after you take each dose.

\section{First Dose}

Take your first dose. It will take 30 to 45 minutes for this medicine to start working.

\section{Second Dose}

About 1 hour after your first dose, check to see how you feel. Remember, only take your second dose if you feel better, about the same, or not much worse.

\section{Third Dose}

Wait 1 hour after your second dose before you take your third dose. This is your last dose until you go to your community clinic appointment.

\section{What else do I need to know?}

When you start taking buprenorphine/naloxone, it usually takes 2 to 3 days to get the right dose of this medicine into your body. Your body will be ready for a higher dose of this medicine tomorrow. Until then, you will likely have some cravings and withdrawal symptoms.

While you're taking buprenorphine/naloxone:

- Don't use another type of opioid ("dope" or "down") such as heroin, fentanyl, morphine, dilaudid, hydromorphone, Percocet, or methadone. If you use other opioids while you're taking buprenorphine/naloxone, you may overdose. If you choose to use other opioids, use as little as possible to lower the risk of an overdose.

- Don't take it with other medicines that make you sleepy or relaxed such as benzodiazepines ("benzos"), alcohol, sedatives, or sleep aids.

- Arrange for someone to drive you home from the hospital after you take this medicine.

- Be careful doing any activity where you need to be alert.

- Stop taking it if it makes you feel worse, sleepy, or you have a hard time focusing.

If you have side effects that you're worried about after you leave the Emergency Department, call Health Link at 811, go back to the Emergency Department, or call 911 for help.

For 24/7 nurse advice and general health information call Health Link at 811.

Current as of: May 17, 2019

Author: Emergency Strategic Clinical Network, Alberta Health Services

This material is for information purposes only. It should not be used in place of medical advice, instruction, or treatment. If you have questions, talk with your doctor or appropriate healthcare provider. This information may be printed and distributed without permission for non-profit, education purposes. The content on this page may not be changed without consent of the author. Contact feedback@myhealth.alberta.ca. 


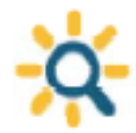

\section{MyHealth.Alberta.ca}

\section{Emergency Department Buprenorphine/Naloxone (Suboxone)}

\section{Home Dosing Information}

The Emergency Department gave you buprenorphine/naloxone (Suboxone) to help treat an opioid use disorder. It's important for you to take this medicine.

You were given 6 doses of buprenorphine/naloxone to take with you.

\section{What is buprenorphine/naloxone and how does it work?}

Buprenorphine/naloxone (Suboxone) is a pill that has 2 medicines. The goal of this medicine is to help you use other opioids less, so you are safer and healthier.

Buprenorphine is a type of opioid (like morphine, heroin or methadone) but it works differently than other types of opioids. Buprenorphine helps treat withdrawal and can get rid of cravings more safely than other medicines. Once you get the right dose of buprenorphine, it will prevent withdrawal symptoms and can help you stop taking other prescription or purchased opioids.

Naloxone (Narcan) is added to buprenorphine to prevent people from injecting it. When the pill is dissolved under the tongue, the naloxone doesn't do anything. If the pill is injected, the naloxone can cause withdrawal symptoms or prevent opioids from working.

\section{How do I take buprenorphine/naloxone?}

Take buprenorphine/naloxone by mouth and dissolve it under your tongue. This medicine may take 15 to 30 minutes to dissolve. It's important to dissolve this medicine under your tongue as it won't work if you swallow it. After you take this medicine, wait at least 30 minutes before you eat or drink.

\section{When should I take buprenorphine/naloxone at home?}

Take this medicine only when you are in moderate withdrawal. This is the point when you feel like you want to use or inject to prevent more withdrawal symptoms.

It's important that you feel sick before you take your first dose of buprenorphine/naloxone. If you take it before you feel really sick, it can make your symptoms much worse.

Before you take your first dose, wait at least:

12 hours since you last used a short-acting opioid (fentanyl, heroin, crushed OxyContin, Percocet).

24 hours since you last used a long-acting opioid (OxyContin taken orally, Hydromorph Contin, OxyNeo).

72 hours since you last used methadone. 


\section{Dosing}

Each dose of buprenorphine/naloxone is $2 \mathrm{mg}$. Be sure to wait at least 1 hour in between doses.

Record the time you take each dose on a piece of paper or in the notes app on your phone.

Don't take your next dose if you feel worse, sleepy, sedated, or have a hard time focusing. If this happens, call the clinic as your dose may be too strong.

You should feel a little better or about the same after you take each dose. As long as you don't feel much worse, you can take your next dose.

\section{First Dose}

Take your first dose. It will take 30 to 45 minutes for the medicine to start working.

\section{Second Dose}

About 1 hour after your first dose, check to see how you feel. Remember, only take your second dose if you feel better, about the same, or not much worse.

\section{Third Dose}

Wait at least 1 hour after the second dose before you take your third dose.

\section{Fourth Dose}

Wait at least 1 hour after the third dose before you take your fourth dose.

\section{Fifth Dose}

Wait at least 1 hour after the fourth dose before you take your fifth dose.

\section{Sixth Dose}

Wait at least 1 hour after the fifth dose before you take your sixth dose. This is your final dose until your community clinic appointment.

\section{While you're taking buprenorphine/naloxone:}

- Don't use another type of opioid ("dope" or "down") such as heroin, fentanyl, morphine, dilaudid, hydromorphone, Percocet, or methadone. If you use other opioids while you're taking buprenorphine/naloxone, you may overdose. If you choose to use other opioids, use as little as possible to lower the risk of an overdose.

- Don't take it with other medicines that make you sleepy or relaxed such as benzodiazepines ("benzos"), alcohol, sedatives, or sleep aids.

- Be careful doing any activity where you need to be alert.

- Stop taking it if it makes you feel worse, sleepy, or you have a hard time focusing.

If you have side effects that you're worried about after you leave the Emergency Department, call Health Link at 811, go back to the Emergency Department or call 911 for help.

For 24/7 nurse advice and general health information call Health Link at 811. 


\begin{tabular}{|c|c|}
\hline \multicolumn{2}{|c|}{ Appendix D: Sites with Implemented Protocol at Time of Publication by Zone } \\
\hline 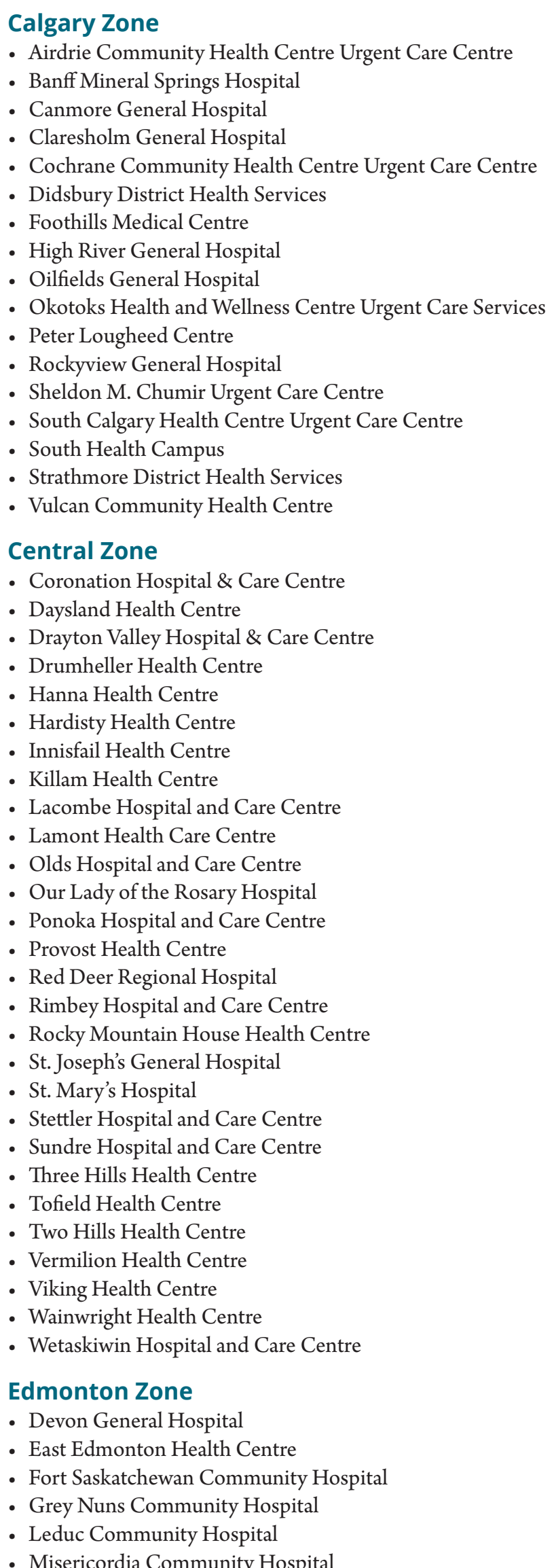 & $\begin{array}{l}\text { - North East Community Health Centre } \\
\text { - Royal Alexandra Hospital } \\
\text { - Stony Plain Westview Health Centre } \\
\text { - Strathcona Community Hospital } \\
\text { - Sturgeon Community Hospital } \\
\text { - University of Alberta Hospital } \\
\text { North Zone } \\
\text { - Athabasca Healthcare Centre } \\
\text { - Barrhead Healthcare Centre } \\
\text { - Beaverlodge Municipal Hospital } \\
\text { - Bonnyville Healthcare Centre } \\
\text { - Boyle Healthcare Centre } \\
\text { - Central Peace Health Complex } \\
\text { - Cold Lake Healthcare Centre } \\
\text { - Edson Healthcare Centre } \\
\text { - Elk Point Healthcare Centre } \\
\text { - Fairview Health Complex } \\
\text { - George McDougall - Smoky Lake Healthcare Centre } \\
\text { - Grande Cache Community Health Complex } \\
\text { - Grimshaw/Berwyn \& District Community Health Centre } \\
\text { - High Prairie Health Complex } \\
\text { - Hinton Healthcare Centre } \\
\text { - La Crete Community Health Centre } \\
\text { - Manning Community Health Centre } \\
\text { - Mayerthorpe Healthcare Centre } \\
\text { - Northern Lights Regional Health Centre } \\
\text { - Northwest Health Centre } \\
\text { - Peace River Community Health Centre } \\
\text { - Redwater Health Centre } \\
\text { - Sacred Heart Community Health Centre } \\
\text { - Seton - Jasper Healthcare Centre } \\
\text { - Slave Lake Health Centre } \\
\text { - St. Theresa General Hospital } \\
\text { - St. Therese - St. Paul Healthcare Centre } \\
\text { - Swan Hills Healthcare Centre } \\
\text { - Wabasca - Desmaris Healthcare Centre } \\
\text { - Westlock Healthcare Centre } \\
\text { - Whitecourt Healthcare Centre } \\
\text { - William J. Cadzow - Lac La Biche Healthcare Centre } \\
\text { South Zone } \\
\text { - Bassano Health Centre } \\
\text { - Big Country Hospital } \\
\text { - Bow Island Health Centre } \\
\text { - Brooks Health Centre } \\
\text { - Cardston Health Centre } \\
\text { - Chinook Regional Hospital } \\
\text { - Coaldale Health Centre } \\
\text { - Crowsnest Pass Health Centre } \\
\text { - Fort Macleod Health Centre } \\
\text { - Medicine Hat Regional Hospital } \\
\text { - Milk River Health Centre } \\
\text { - Pincher Creek Community Health Centre } \\
\text { - Taber Health Centre } \\
\text { - }\end{array}$ \\
\hline
\end{tabular}

